\title{
Predicting Diabetic Foot Maturing Through Evolutionary Computation
}

\author{
José Neves (D), André Alves (D), Marco Prata (D), Mário Ribeiro (D), \\ Victor Alves (D), Filipa Ferraz $\mathbb{D}^{D}$, João Neves $(\mathbb{D}$, Jorge Ribeiro $(\mathbb{D}$, \\ António Capita (D) and Henrique Vicente (D)
}

\begin{abstract}
It is a twenty-first-century disease, its numbers are still growing exponentially. This brings one to the subject of this work, the Maturing of Diabetic Foot which, like diabetes, rises to values never seen before. It is envisaging the development of an ImageJ plug-into extract relevant feature from diabetic foot images and, in conjunction with the patient's clinical and lifelong data, a computational system to
\end{abstract}

J. Neves $\cdot$ V. Alves $\cdot$ F. Ferraz $\cdot$ H. Vicente $(\bowtie)$

Centro Algoritmi, Universidade do Minho, Braga, Portugal

e-mail: hvicente@uevora.pt

J. Neves

e-mail: jneves@di.uminho.pt

V. Alves

e-mail: valves@di.uminho.pt

F. Ferraz

e-mail: filipatferraz@gmail.com

A. Alves $\cdot$ M. Prata $\cdot$ M. Ribeiro

Departamento de Informática, Escola de Engenharia, Universidade do Minho, Braga, Portugal e-mail: a69381@alunos.uminho.pt

M. Prata

e-mail: a68516@alunos.uminho.pt

M. Ribeiro

e-mail: a67657@alunos.uminho.pt

J. Neves

Mediclinic Arabian Ranches, 282602, Dubai, United Arab Emirates

e-mail: joaocpneves@gmail.com

J. Ribeiro

Escola Superior de Tecnologia e Gestão, ARC4DigiT-Applied Research Center for Digital Transformation, Instituto Politécnico de Viana do Castelo, Viana do Castelo, Portugal e-mail: jribeiro@estg.ipvc.pt

\section{A. Capita}

Instituto Superior Técnico Militar, Luanda, Angola

e-mail: antoniojorgecapita@gmail.com

(C) Springer Nature Singapore Pte Ltd. 2019

109

S.-L. Peng et al. (eds.), Computing and Network Sustainability, Lecture Notes in Networks and Systems 75, https://doi.org/10.1007/978-981-13-7150-9_11 9 pp. 
predict and evaluate its severity. The applied problem-solving method is based on a symbolic/sub-symbolic line of logical formalisms that make complex systems easier to develop and analyze, where solutions to new problems are based on answers to previous ones, and itemized as a Case-Based Reasoning/Artificial Neural Network approach to computing.

Keywords Diabetic mellitus $\cdot$ Case-based reasoning $\cdot$ Artificial neural networks • Image $\cdot$ Many-valued machines

H. Vicente

Departamento de Química, Escola de Ciências e Tecnologia, Centro de Química de Évora, Universidade de Évora, Évora, Portugal 